\title{
Time-clustering investigation of fire temporal fluctuations in Portugal
}

\author{
L. Telesca ${ }^{1}$ and M. G. Pereira ${ }^{2,3}$ \\ ${ }^{1}$ Istituto di Metodologie per l'Analisi Ambientale, CNR, Tito (PZ), Italy \\ ${ }^{2}$ Centre for Research and Technology of Agro-Environment and Biological Sciences (CITAB), University of Trás-os-Montes \\ and Alto Douro, Vila Real, Portugal \\ ${ }^{3}$ Departamento de Física, Escola de Ciência e Tecnologia, Universidade de Trás-os-Montes e Alto Douro (DF-ECT UTAD), \\ Vila Real, Portugal
}

Received: 14 August 2009 - Revised: 11 January 2010 - Accepted: 25 January 2010 - Published: 6 April 2010

\begin{abstract}
Temporal clustering structures were identified and quantified in fire sequences recorded from 1980 to 2005 in Continental Portugal, by using the Allan Factor statistics, a statistical tool suited to reveal clustering behaviour in point processes. The obtained results show the presence of daily and annual periodicities, superimposed onto a scaling behaviour, which features the sequence of wildfires as a fractal time process with a rather high degree of time-clusterization of the events.
\end{abstract}

\section{Introduction}

According to Joint Research Centre of the European Commission (Barbosa et al., 2007), the countries of the northern arch of the Mediterranean Sea (Portugal, Spain, France, Italy and Greece) are the European countries mostly affected by wildfires. Portugal is particularly affected and presents the highest number of fires $(N)$ and the third highest burnt area (A) absolute value, with almost the same amount as Italy, the second most affected country.

Pereira et al. (2005) analyse the spatial and temporal variability of forest fire activity and the synoptic patterns associated with air masses favourable to high wildfire activity in Portugal and conclude that, i) about $80 \%$ of total $A$ is due to a small proportion of fires during $10 \%$ of the summer days characterised by specific and non-frequent atmospheric circulation pattern, ii) both the inter- and the intra-annual variabilities are related to meteorological anomalous dry conditions before and during the summer fire season.

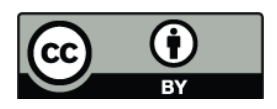

Correspondence to: M. G. Pereira (gpereira@utad.pt)
The relation between high wildfire activity and specific weather/climate conditions was also emphasized during the exceptional fire season of 2003, with total burnt area of 450000 ha (about $5 \%$ of Portugal mainland), when the vast majority of the burnt area by wildfires in Continental Portugal was registered during a heatwave, characterised by extreme values of several meteorological fields, namely exceptionally high values of maximum and minimum temperatures, particularly low humidity levels and relatively high wind speed (Trigo et al., 2005, 2006).

Weather controls (directly or indirectly) other aspects/factors of wildfire activity such as the availability/existence and state of the vegetation and dead fuels as well as fire behaviour. On the other hand, periods of favourable weather conditions to the occurrence and development of (large) wildfires are not very frequent and have a typical duration of a few days to one or two weeks. In this sense, it could be expected that a time-concentration of the wildfire occurrences in specific periods.

The objective of this work is to investigate the existence of time-clustering behaviour in the fire dynamics and the presence of time-correlation structures in the time distribution of the fire events in Continental Portugal. We intend to apply the Allan Factor, which is well suited to detect scaling behaviour in the point processes.

In fact, a sequence of fires can be considered as a point process, where events are characterised by spatial location and occurrence time and marked by the size of burnt area. Dynamics of fires are well described by using the concept of self-organized critical (SOC) systems (Clar et al., 1999; Drossel and Schwabl, 1992, 1993; Honecker and Peschel, 1997). The SOC idea explains complex dynamical behaviours in many natural phenomena, which are represented

Published by Copernicus Publications on behalf of the European Geosciences Union. 
as extended dynamical systems operating at states of critical equilibrium: the only spatial or temporal scales are those deduced from the size of the system (Bak et al., 1988). The main features of SOC systems are spatial fractality, temporal fluctuations scaling as $1 / f^{\alpha}$ and the power-law frequency distribution of sizes. In the context of studies concerning fires, much attention has been focused on the problem of the frequency distribution of burnt areas, leading to the more or less complex models fitting real observations (Malamud et al., 1998; Ricotta et al., 1999, 2001; Song et al., 2001; Reed and McKelvey, 2002). All these studies deal with one of the parameters that define a fire event, i.e. its intensity given by size of burnt area. But the concept of self-organized criticality also implies that the time evolution of the system is time-scaling. Recently, several papers have been focused on the temporal fluctuations of fire sequences, revealing the presence of scaling behaviour in the time dynamics of fire (Telesca et al., 2005; Corral et al., 2008) through the presence of the power-law shape in the statistics describing the temporal distribution of fires.

A comprehensive characterisation of the temporal clustering structures of the fire sequences, along with all the other aspects wildfire activity contributes to, characterise the fire regime in continental Portugal. To continue, a brief description of the data used in this work and a concise characterisation of the study area performed is in Sect. 2. Definitions and characteristics of the methodology are discussed in Sect. 3, while the results are presented in Sect. 4. Section 5 is devoted to discussion and conclusions.

\section{Study area and dataset}

Portugal holds the largest wildfire land datasets in the European context, with more than 450000 records in the 19802005 period. This is only possible because of the work of the National Forestry Authority (Autoridade Florestal Nacional, AFN) that systematically compile pertinent information about each wildfire occurring in continental Portugal since 1980 and kindly provides access to the dataset. The fire data only relies on in situ information and includes information about the fire location, type (single or re-ignition), date and time of ignition and extinction. A small number $(<2.7 \%)$ of data and format errors in the dataset was identified and corrected. The sum of the burnt area by all the fires in this dataset is equal to $3.0 \times 10^{6}$ ha of burnt area, which represents $34 \%$ of continental Portugal area. However, with this dataset, it is not possible to demonstrate if the same area might have been burnt by wildfires more than once (Pereira et al., 2006).

Portugal is the south-westernmost country of mainland Europe and is bordered by the Atlantic Ocean to the west and south and by Spain to the north and east (Fig. 1). According to the Köppen classification scheme, Portugal climate can be classified as Mediterranean type csa in the southern ar- eas, and $c s b$ in the north (Peel et al., 2007). Portugal is one of the warmest European countries with a distinct seasonal climate, characterised by mild and rainy winters and warm and dry summers (Trigo and DaCamara, 2000; Meteorological Institute, 2008). In general, vegetation is well adapted to the difference in rainfall and temperature between winter and summer seasons but these weather/climate conditions are quite favourable for the occurrence of wildfires during the summer season. The total monthly values of the number of fires and the amount of burnt area (Fig. 2) illustrates this fact. Wildfire activity is not spread homogeneously over the entire country. The central and northern mainland are the preferred areas for fire activity in Portugal because this part of the territory have more irregular topography, a more dense hidrological network, and more fire prone ecosystems.

\section{Method}

A sequence of fire events can be considered as a realization of a stochastic temporal point process, which describes events that occur at some random locations in time (Cox and Isham, 1980). A point process is fractal when a number of its relevant statistics exhibit scaling with related scaling exponents, that indicate the represented phenomenon contain clusters of points over a relatively large set of time scales (Lowen and Teich, 1996, 1999).

The well-known method to investigate the temporal properties of a time series is the power spectral density (PSD) $S(f)$, obtained by means of a Fourier Transform (FT) of the series. The PSD gives information on how the power of the series is concentrated at various frequency bands. This information allows identifying periodic, multi-periodic or nonperiodic frequency patterns. Usually the power spectrum plotted in log-log scales is used to analyse broadband behaviour. The power-law (or fractal) dependence (linear on a log-log plot) of the PSD is given by $S(f) \sim f^{-\alpha}$ and is a hallmark of the presence of time-scaling in the data. The properties of the series can be further classified in terms of the numerical value of the spectral (or fractal) exponent: $\alpha=0$ features white noise time series, characterised by the absence of time correlations; while $\alpha \neq 0$ is typical of pink noise time series, characterised by the presence of time correlations.

A fire sequence is a temporal point process and the simple application of the FT is not possible. In order to be able to analyse the temporal fluctuations of fires, these can be represented by a finite sum of Dirac's delta functions centred on the occurrence times $t_{i}$, with amplitude $A_{i}$ proportional to the burnt area:

$y(t)=\sum_{i=1}^{N} A_{i} \delta\left(t-t_{i}\right)$,

where $N$ represents the number of events recorded. 

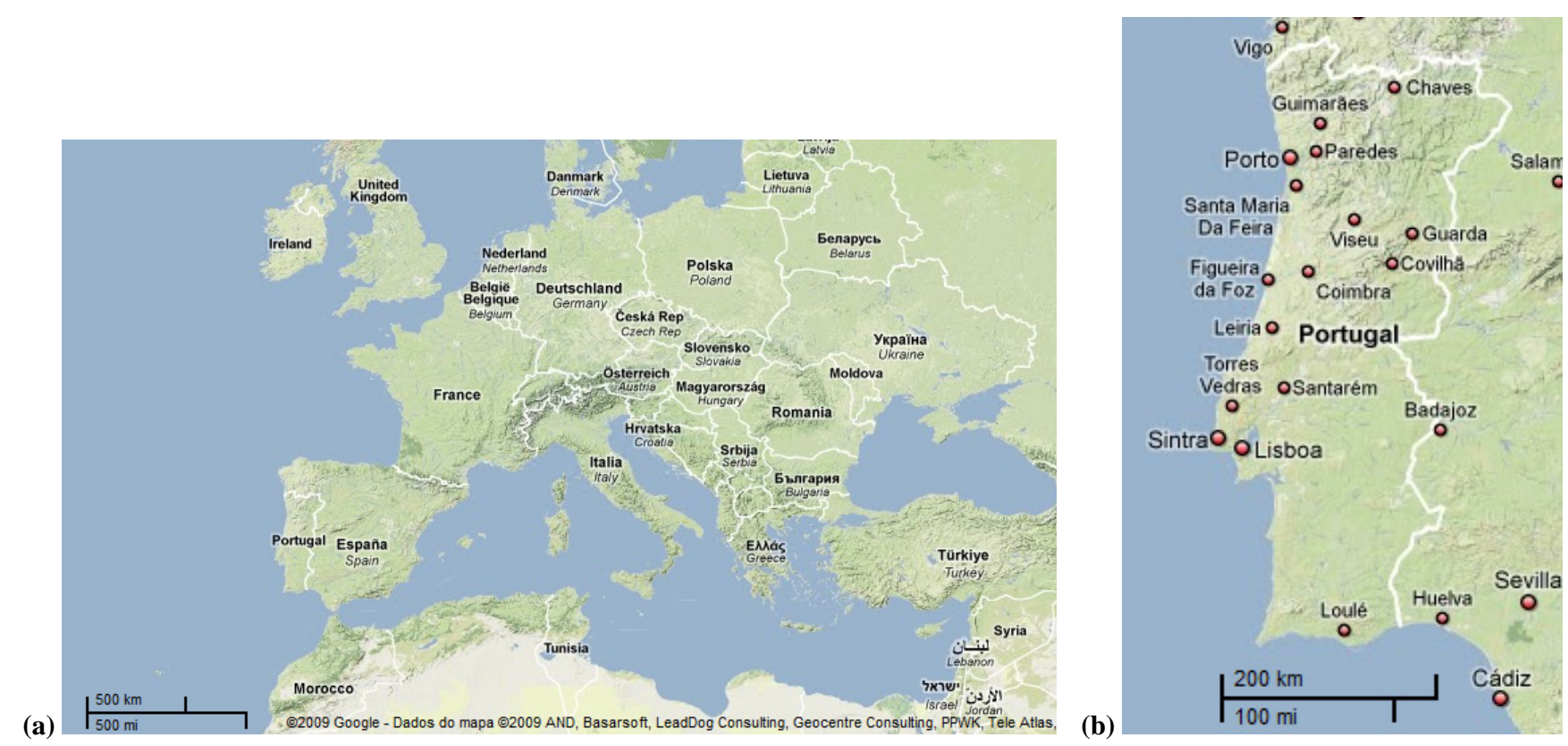

Fig. 1. (a) Map of Europe and North Africa, retrieved from Google Maps website http://maps.google.pt/, and (b) a zoom of Portugal for a better visualization of the country orography.

Then, dividing the time axis into equally spaced contiguous counting windows of duration $\tau$, which is called timescale, a sequence of counts $\left\{N_{k}(\tau)\right\}$ is produced, with $N_{k}(\tau)$ denoting the number of fire events in the $k$-th window (Lowen and Teich, 1999):

$N_{k}(\tau)=\int_{t_{k}}^{t_{k+\tau}} \sum_{j=1}^{n} \delta\left(t-t_{j}\right) d t$.

The following quantity, the Allan Factor (AF), can be defined as

$\operatorname{AF}(\tau)=\frac{<\left(N_{k+1}(\tau)-N_{k}(\tau)\right)^{2}>}{2<N_{k}(\tau)>}$

which is related to the variability of successive counts (Turcott et al., 1994; Allan, 1966); the symbol $<\ldots>$ indicates the average value. The AF has been largely used to investigate the time dynamics of earthquakes (Telesca et al., 2004).

If the point process is time-correlated, then the AF varies with the timescale $\tau$ with a power-law form:

$\mathrm{AF}(\tau)=1+\left(\frac{\tau}{\tau_{1}}\right)^{\alpha}$

over a certain range of timescales $\tau$; and the exponent $\alpha$ quantifies the strength of time-correlation; $\tau_{1}$ is the fractal onset time and marks the lower limit for significant scaling

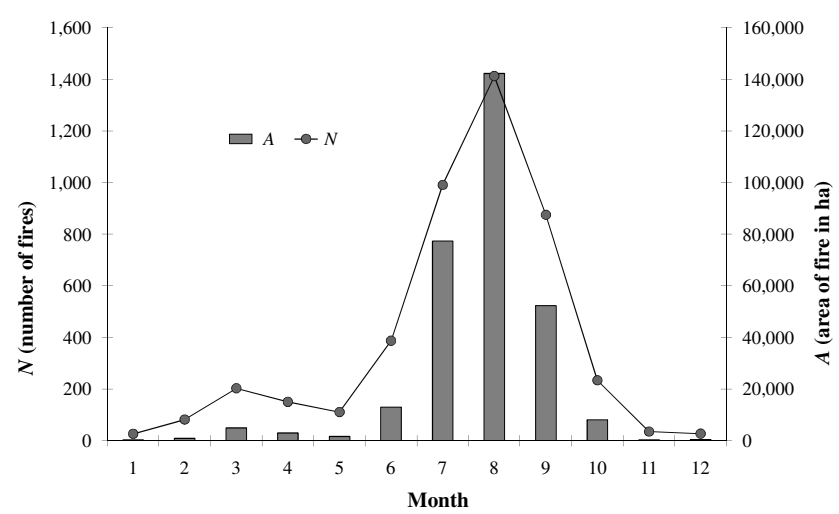

Fig. 2. Monthly average of burnt area, $A$, (grey bars) and of number of fire occurrences, $N$, (solid line) in continental Portugal for the 1980-2005 period.

behaviour in the AF, so that for $\tau \ll \tau_{1}$ the time-scaling property becomes negligible at these time scales (Lowen and Teich, 1996). The AF statistic assumes values near unity for Poisson processes (Thurner et al., 1997). Therefore, if $\alpha=0$ the point process is Poissonian, which means that the series is memoryless and constituted by independent events; while if $\alpha>0$ the process is characterised by time-scaling behaviour, which means that the series is time-correlated. 


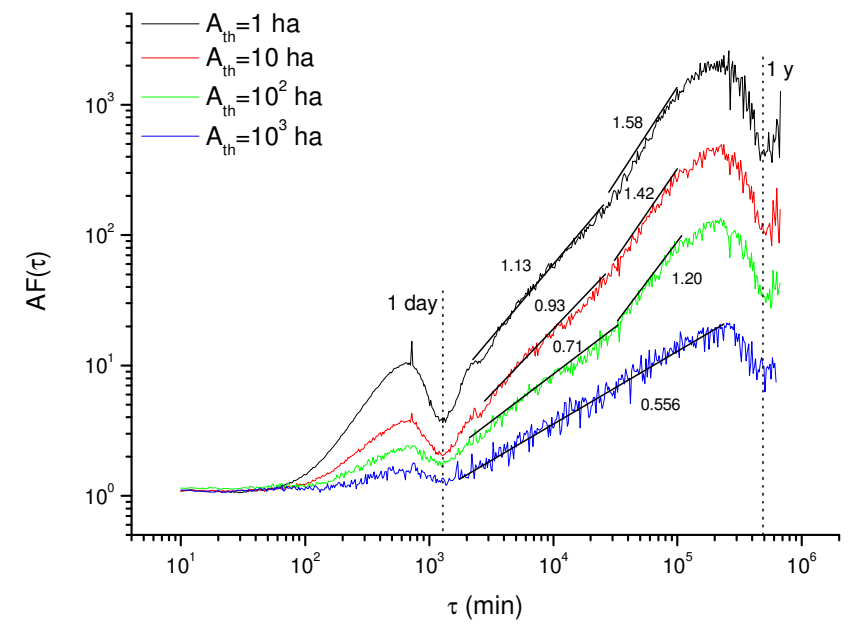

Fig. 3. Log-log plot of the Allan Factor (AF) as a function of the timescale $\tau$, for the Portuguese fire series, considering fire events with burnt area $(A)$ above or equal increasing thresholds $\left(A_{\text {th }}\right)$ of 1 , $10,10^{2}$ and $10^{3}$ ha, registered in the 1980-2005 period.

\section{Results}

During the observation period, about 450000 forest fires have been recorded. Figure 3 shows the relation $\operatorname{AF}(\tau) \sim$ $\tau$ in $\log -\log$ scales for the Portuguese fire sequences for timescales $\tau$ ranging between $10 \mathrm{~min}$ and $T / 10$, where $T$ is the total observation period in minutes of the fire catalogue. The analysis was performed for several threshold burnt areas $A_{\text {th }}$ and considering, in each case, the events with burnt area $A \geq A_{\text {th }}$. The number of fire events and the obtained scaling exponent values for the intermediate and higher timescales ranges in each analysis are presented in Table 1 . The following other results were obtained:

- The fire activity in Portugal is not Poissonian, because the AF curves are not flat for all the timescales $\tau$. This result indicates the presence of time-clustering behaviour in the fire dynamics, which implies the presence of time-correlation structures in the time distribution of the fire events.

- The time dynamics of the fire sequences are characterised by the presence of periodicities, shown by the drops in the AF curves (indicated by vertical dotted lines in Fig. 3). The periodic component at about 1 day is clearly related with the daily meteorological cycle. Pippen (1999) and Plucinski (1999) found a diurnal variation in the state of the dead-fuel moisture content of open woodland fuel, in response to the normal diurnal weather changes, such as the rise and fall of temperature and solar radiation. The component at about 1 year is connected with the yearly cycle of vegetation, induced by the meteo-climatic yearly cycle. In particular
Table 1. Number $(N)$ and scaling exponent $(\alpha)$ of fire sequences with burnt area $(A)$ above or equal a given burnt area threshold $\left(A_{\mathrm{th}}\right), N\left(A \geq A_{\mathrm{th}}\right)$, for increasing values of the threshold $A_{\mathrm{th}}$. Regarding the scaling exponent, the first value corresponds to the intermediate timescale range, the second value to the higher timescale range.

\begin{tabular}{lcccc}
\hline$A_{\text {th }}$ & 1 ha & 10 ha & $10^{2}$ ha & $10^{3}$ ha \\
\hline$N$ & 138935 & 20607 & 4106 & 444 \\
& $1.13 \pm 0.01$ & $0.93 \pm 0.01$ & $0.71 \pm 0.01$ & $0.556 \pm 0.006$ \\
$\alpha$ & $1.58 \pm 0.02$ & $1.42 \pm 0.03$ & $1.20 \pm 0.03$ & \\
\hline
\end{tabular}

the evidenced periodicities are more powerful for lower threshold burnt areas, as revealed by the "deeper" drops in the AF curves.

- All the AF curves are flat at small timescales up to a cutoff timescale of order $102 \mathrm{~min}$. In particular, the cutoff timescale, which indicates the smallest timescale from which the scaling appears visible, seems to increase with the increase of the threshold burnt area $A_{\text {th }}$.

- In the intermediate timescale range, two scaling regions (given by the linear parts of the log-log AF curve in Fig. 3) could be identified in the AF curves $\left(A_{\text {th }}=1\right.$ ha, $10 \mathrm{ha}$ and $10^{2} \mathrm{ha}$ ), while only one scaling region is visible in the AF curve for $A_{\mathrm{th}}=10^{3}$ ha. The crossover timescale between the two scaling regions is about 20 days: the origin is not well clear and might be due to mixed natural and anthopogenic causes. The scaling exponents, which reveal the strength of the clusterization of the events, decrease, in both the regions, with the increase of the threshold burnt area $A_{\text {th }}$. This indicates that the sequences tend toward Poissonian behaviour with the increase of the threshold burnt area.

- The obtained results are very similar to those obtained with other fire datasets in Italy (Telesca and Lasaponara, 2006) and Argentina (Ghermandi et al., 2008).

\section{Discussion and conclusions}

The fire sequences recorded in Portugal were analysed in order to characterise their time dynamics. The applied AF methodology has revealed the presence of several time regimes (periodicities and scaling regimes) mainly connected with meteorological and climatic conditions of the area.

The presence of scaling regimes in the AF statistics evidences the self-organization of the fire activity: fires have a direct impact on vegetation, which, in turn, contributes to the future fire activity, therefore, the existence of feedback 
mechanisms involving both fires and the ecological patterns (vegetation type, age, physiognomy, etc.) gives rise to correlation structures and memory phenomena, revealed by the time-scaling behaviour in the AF statistics.

The vegetation patterns constrain and, at the same time, are constrained by the processes that generate them. A fire occurring in an area, which was never burned before, creates a pattern of burnt and unburnt vegetation patches, which will influence the occurrence and size of the next fire within a continuous feedback dynamics. In fact, a negative correlation has been found between the expected fire-size and the existence and abundance of recently disturbed areas by Cumming (2001) using a parametric statistical model developed during the analysis of the fire-size distribution of fires in the boreal mixedwood forests of Canada.

The presence of different scaling regimes indicates that different dynamical mechanisms govern the same sequence and different values of the scaling exponent $\alpha$ suggest different strengths of the power-law fluctuations of the fire sequence. The different mechanisms could be due to mixed natural and anthropogenic causes. This knowledge can be used by the forest managers to change fire behaviour and increase forest resistance to fire.

The result seems to indicate that large wildfires are less dependent on these mechanisms than small and medium size wildfires $\left(A_{\mathrm{th}} \leq 10^{2} \mathrm{ha}\right)$, since a single scaling region characterises larger fires. Periodicities are also less evident for higher burnt area threshold which may be a sign that large wildfires are much less dependent on the daily and annual (weather/vegetation) cycles than the small- and medium-size fires which confirm the occurrence of large wildfires is essentially dependent on the existence of extreme fire weather conditions.

Acknowledgements. The authors want to express their gratitude to the Autoridade Florestal Nacional, (AFN), the Portuguese National Forestry Authority, for kindly providing the fire data to perform this work.

Edited by: R. Lasaponara

Reviewed by: M. Lovallo and two other anonymous referees

\section{References}

Allan, D. W.: Statistics of Atomic Frequency Standards, Proc. IEEE, 54, 221-230, 1966.

Bak, P., Tang, C., and Wiesenfeld, K.: Self-organized criticality, Phys. Rev. A, 38, 364-374, 1998.

Barbosa, P., Amatulli, G., Boca, R., Camia, A., Kucera, J., Libertà, G., San-Miguel Ayanz, J., Schmuck, G., Schultem, E., and Dierksm, H.-H.: Forest Fires in Europe 2006, EUR 22931 EN Joint Research Centre - Institute for Environment and Sustainability, Luxembourg: Office for Official Publications of the Eu- ropean Communities, EUR - Scientific and Technical Research series, ISSN 1018-5593, 2007.

Clar, S., Drossel, B., Schenk, K., and Schwabl, F.: Self-organized criticality in forest-fire models, Physica A, 266, 153-159, 1999.

Corral, A., Telesca, L., and Lasaponara, R.: Scaling and correlations in the dynamics of forest-fire occurrences, Phys. Rev. E, 77, 016101, doi:10.1103/PhysRevE.77.016101, 2008.

Cox, D. R. and Isham, V.: Point Processes, London: Chapman and Hall, 1980.

Cumming, S. G.: Parametric models of the fire size distribution, Can J. Forest Res., 31, 1297-1303, 2001.

Drossel, B. and Schwabl, F.: Self-Organized Criticality and Synchronization in a Forest-Fire Model, Phys. Rev. Lett., 69, 16291632, 1992.

Drossel, B. and Schwabl, F.: Self organization in a forest-fire model, Fractals, 1, 1022-1029, 1993.

Ghermandi, L., de Torres Curth, M., Franzese, J., and Telesca, L.: Analysis of time regimes in fire sequences occurred in Patagonia, Argentina, Fluct. Noise Lett., 8, L175-L181, 2008.

Honecker, A. and Peschel, I.:. Length scales and power laws in the two-dimensional forest-fire model, Physica A, 239, 509-530, 1997.

Lowen, S. B. and Teich, M. C.: The Periodogram and Allan Variance Reveal Fractal Exponents Greater than Unity in AuditoryNerve Spike Trains, J. Acoust. Soc. Am., 99, 3585-3591, 1996.

Lowen, S. B. and Teich, M. C.: Estimation and Simulation of Fractal Stochastic Point Processes, Fractals, 3, 183-210, 1999.

Malamud, B. D., Morein, G., and Turcotte, D. L.: Forest fires: An example of self-organized critical behaviour, Science, 281, 1840-1842, 1998.

Meteorological Institute: Climate Profile, Mainland Portugal, available at: www.meteo.pt/resources/im/pdfs/clim_ac_00_00_ en.pdf(last access: 4 March 2008), 2008.

Peel, M. C., Finlayson, B. L., and McMahon, T. A.: Updated world map of the Köppen-Geiger climate classification, Hydrol. Earth Syst. Sci., 11, 1633-1644, 2007, http://www.hydrol-earth-syst-sci.net/11/1633/2007/.

Pereira, M. G., Trigo, R. M., DaCamara, C. C., Pereira, J. M. C., and Leite, S. M.: Synoptic patterns associated with large summer forest fires in Portugal, Agr. Forest Meteorol., 129, 11-25, 2005.

Pereira, J. M. C., Carreiras, J. M. B., Silva, J. M. N., and Vasconcleos, M. J.: Alguns Conceitos Básicos Sobre os Fogos Rurais em Portugal, in: Incêndios Florestais em Portugal, ISA Press, Lisboa, Portugal, 133-161, 2006.

Pippen, B. G.: Predicting fine fuel moisture in shrubby vegetation, Honours thesis, Australian National University, 1999.

Plucinski, M. P.: The investigation of factors governing ignition and development of fires in heatland vegetation, Ph.D. thesis, University of New South Wales, ADFA, 1999.

Reed, W. J. and McKelvey, K. S.: Power-law behaviour and parametric models for the size-distribution of forest fires, Ecol. Model., 150, 239-254, 2002.

Ricotta, C., Arianoutsou, M., Diaz-Delgado, R., Duguy, B., Lloret, F., Maroudi, E., Mazzoleni, S., Moreno, J. M., Rambal, S., Vallejo, R., and Vazquez, A.: Self-Organized criticality of wildfires ecologically revisited, Ecol. Model., 141, 307-311, 2001.

Ricotta, C., Avena, G., and Marchetti, M.: The flaming sandpile: self-organized criticality and wildfires, Ecol. Model., 119, 73$77,1999$. 
Song, W., Weicheng, F., Bighong, W., and Jianjun, Z.: Selforganized criticality of forest fire in China, Ecol. Model. 145, 61-68, 2001.

Telesca, L., Lapenna, V., Lovallo, M., and Macchiato, M.: Longrange time-correlation properties of seismic sequences, Chaos Soliton Fract., 21, 387-393, 2004.

Telesca, L., Amatulli, G., Lasaponara, R., Lovallo, M., and Santulli, A.: Time-scaling propertiesin forest-fire sequences observed in Gargano are (southern Italy), Ecol. Model., 185, 531-544, 2005.

Telesca, L. and Lasaponara, R.: Emergence of temporal regimes in fire sequences, Physica A, 359, 747-751, 2006.

Thurner, S., Lowen, S. B., Feurstein, M. C., Heneghan, C., Feichtinger, H. G., and Teich, M. C.: Synthesis, and Estimation of Fractal-Rate Stochastic Point Processes, Fractals 5, 565-595, 1997.

Trigo, R. M. and DaCamara, C. C.: Circulation Weather Types and their impact on the precipitation regime in Portugal, Int. J. Climatol., 20, 1559-1581, 2000.
Trigo, R. M., García-Herrera, R., Díaz, J., Trigo, I. F., and Valente, M. A.: How exceptional was the early August 2003 heatwave in France?, Geophys. Res. Lett., 32, L10701, doi:10.1029/2005GL022410, 2005.

Trigo, R. M., Pereira, J. M. C., Pereira, M. G., Mota, B., Calado, T. J., DaCamara, C. C., and Santo, F. E.: Atmospheric conditions associated with the exceptional fire season of 2003 in Portugal, Int. J. Climatol., 26(13), 1741-1757, 2006.

Turcott, R. G., Lowen, S. B., Li, E., Johnson, D. H., Tsuchitani, C., and Teich, M. C.: A Nonstationary Poisson Point Process Describes the Sequence of Action Potentials Over Long Time Scales in Lateral-Superior-Olive Auditory Neurons, Biol. Cybern., 70, 209-217, 1994. 